\title{
Measuring the Properties of the Higgs Boson with the ATLAS Detector
}

\author{
W. Quayle on behalf of the ATLAS Collaboration \\ University of Wisconsin-Madison, 1150 University Ave., Madison, WI, 53706
}

\begin{abstract}
If an excess of events is discovered in the Higgs boson searches at LHC, it will be important establish whether the excess is a Higgs boson or a different particle with similar decay modes. This talk reviews the measurements of the Higgs boson properties that are planned for the ATLAS detector at the LHC.
\end{abstract}

Keywords: Higgs

PACS: $14.80 . \mathrm{Bn}, 14.80 . \mathrm{Cp}$

\section{INTRODUCTION}

One of the major goals of the LHC is to understand the mechanism of Electroweak symmetry breaking by searching for evidence of the Higgs boson [1, 2, 3]. The strongest such constraints presently come from searches at LEP, which has excluded a Standard Model Higgs boson with a mass below $114.4 \mathrm{GeV}$ [4], and from Tevatron, which has ruled out the existence of a Standard Model Higgs boson with a mass of $170 \mathrm{GeV}$ [5].

The ATLAS detector [6] at the LHC will either find evidence for a Higgs boson or place strong constraints on its production cross-section that will contradict the prediction of the Standard Model [7]. If a Higgs-like particle is found, its properties must be measured to establish that it is indeed a Higgs boson and not a different particle.

\section{MASS, SPIN, AND CP PROPERTIES OF THE HIGGS BOSON}

Observables such as the Higgs boson's branching ratios vary considerably as a function of mass; in order to interpret the other property measurements, one must measure the Higgs boson mass precisely. The Higgs boson mass determination at ATLAS is dominated by the $H \rightarrow \gamma \gamma$ and $H \rightarrow Z Z \rightarrow 4 \ell$ channels.

The $H \rightarrow \gamma \gamma$ channel is sensitive in the mass range below about $150 \mathrm{GeV}$, and its mass resolution is $1.36 \mathrm{GeV}$ for a Higgs boson with a mass of $120 \mathrm{GeV}$. The $H \rightarrow Z Z \rightarrow 4 \ell$ channel is sensitive for a broad range of Higgs boson masses. Its resolution in the region below the $Z Z$ threshold ranges from $1.8 \mathrm{GeV}$ at $M_{H}=120 \mathrm{GeV}$ to $2.7 \mathrm{GeV}$ at $M_{H}=180 \mathrm{GeV}$. The resolution degrades slightly at higher mass, but for very heavy Higgs bosons the natural width is larger than the experimental resolution. The expected precision of a measurement of the natural width of the Higgs boson after collecting 300 $\mathrm{fb}^{-1}$ of integrated luminosity is better than $8 \%$ for masses above $270 \mathrm{GeV}$ [8]. It is worth noting that the mass resolutions quoted here are taken from very recent studies, but other 

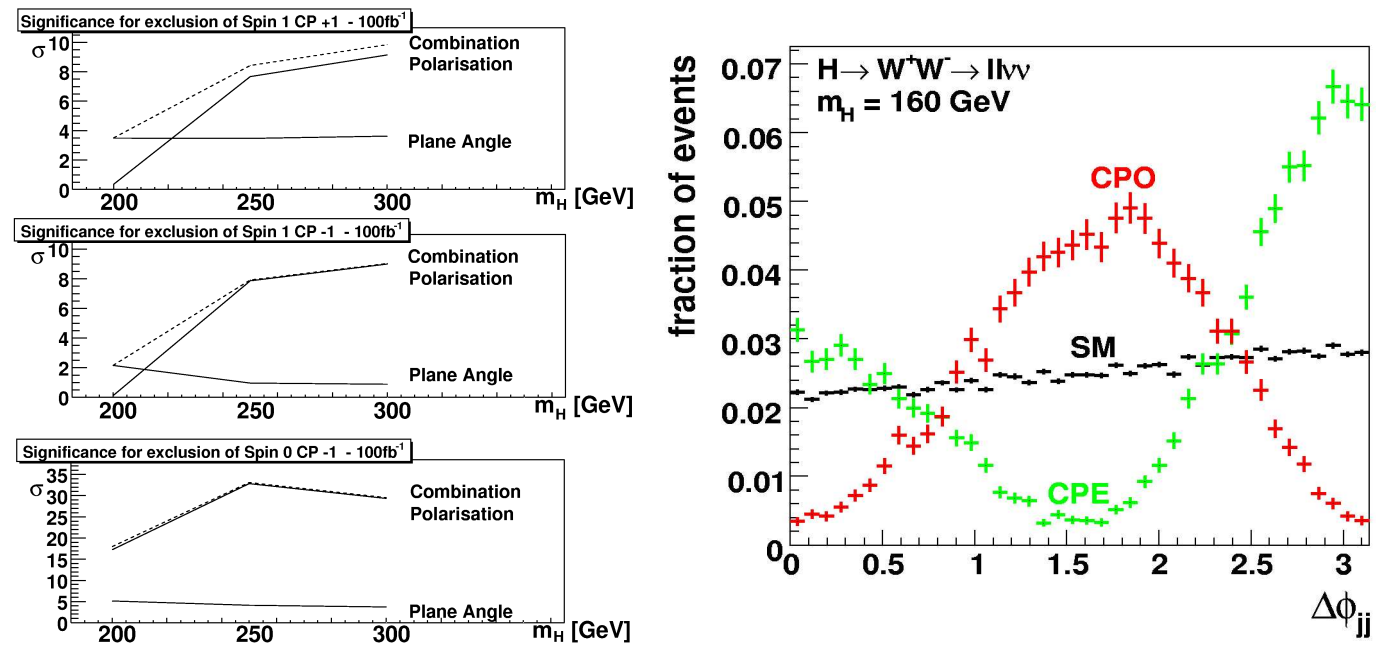

FIGURE 1. Left: the expected significance for the exclusion of several alternative spin/CP scenarios as a function of the Higgs boson mass, after $100 \mathrm{fb}^{-1}$ of integrated luminosity. Right: the transverse opening angle between the two tag jets in the Weak Boson Fusion $H \rightarrow W W \rightarrow \ell v \ell v$ signal, for the Standard Model case (SM, black), a CP-odd scenario (CPO, red), and a CP-even scenario (CPE, green).

measurements, such as spin, $\mathrm{CP}$, and coupling determinations, are based on older studies that have not yet been updated with the latest understanding of the detector.

A spin-1 boson cannot be produced by gluon fusion or decay to a photon pair [9], so if the decay $H \rightarrow \gamma \gamma$ is observed, then the spin of the Higgs boson cannot be 1 . If the decay $H \rightarrow \gamma \gamma$ is not observed, it is still possible to extract information about the spin of the Higgs boson from angular correlations in the $H \rightarrow Z Z \rightarrow 4 \ell$ channel and the angular distributions of the struck quarks in Higgs production by Weak Boson Fusion.

The Higgs boson spin and CP are extracted from $H \rightarrow 4 \ell$ decays using three main observables: $\alpha, \beta$, and $R$ [10]. $\alpha$ and $\beta$ are obtained by fitting $F(\phi)=1+\alpha \cos (\phi)+$ $\beta \cos (2 \phi)$ to the distribution of $\phi$, the angle between the decay planes of the two $Z$ bosons in the rest frame of the Higgs boson. $R$ is given by $R=(L-T) /(L+T)$, where $L$ and $T$ are obtained by fitting $G(\theta)=T\left(1+\cos ^{2}(\theta)\right)+L \sin ^{2}(\theta)$ to the distribution of the angle $\theta$ between the $Z$ boson decay products and the receding Higgs boson in the rest frame of the $Z$ boson. The left plot in Figure 1 shows the significance with which several alternative models can be ruled out with $100 \mathrm{fb}^{-1}$ of integrated luminosity. The analysis of $\theta$ can rule out a non-Standard-Model Higgs boson with a mass larger than about $250 \mathrm{GeV}$; the analysis of $\phi$ through the coefficients $\alpha$ and $\beta$ yields the strongest discriminating power for Higgs bosons with a mass around $200 \mathrm{GeV}$.

Searches for Higgs bosons via Weak Boson Fusion use several cuts on the two highest$P_{T}$ jets in the event ("tag" jets) to achieve a strong rejection against backgrounds [11]. The most sensitive search channels for this production mechanism are $H \rightarrow \tau \tau$ in the region around $M_{H}=120 \mathrm{GeV}$ and $H \rightarrow W W \rightarrow \ell v \ell v$ in the region around $M_{H}=$ $160 \mathrm{GeV}$. One can extract information about the tensor structure of the $H V V$ vertex by analyzing the transverse angle $\Delta \phi_{j j}$ between the two leading jets [12]; the right plot in Figure 1 shows the $\Delta \phi_{j j}$ distribution for $H \rightarrow W W$ signal in the Standard Model and two alternate models. A $\chi^{2}$ test is used to determine the dominant couplings. With 

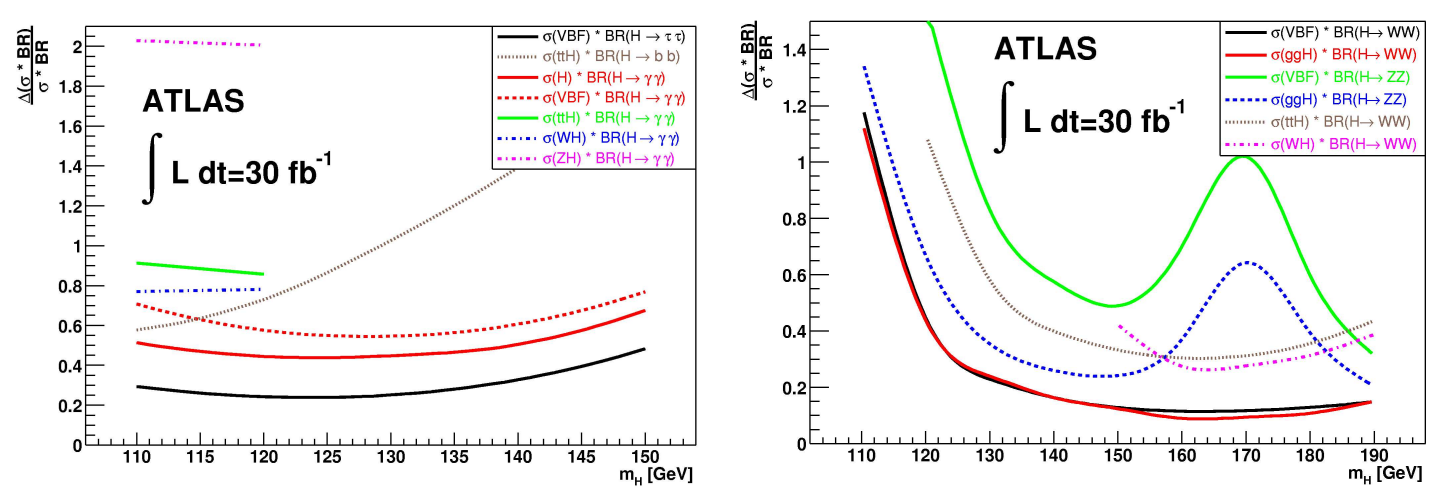

FIGURE 2. The expected relative error (after collecting $30 \mathrm{fb}^{-1}$ of integrated luminosity) on the measurement of the rates for several channels. Left: $H \rightarrow b \bar{b}$ and $H \rightarrow \gamma \gamma$, for $110<M_{H}<150 \mathrm{GeV}$. Right: $H \rightarrow W W$ and $H \rightarrow Z Z$, for $110<M_{H}<150 \mathrm{GeV}$.

$10 \mathrm{fb}^{-1}$ of integrated luminosity, one would expect to exclude the CP-even (CP-odd) hypothesis with a confidence level corresponding to $5.4 \sigma(4.6 \sigma)$ using the $H \rightarrow W W$ channel if the Higgs boson mass is $160 \mathrm{GeV}$. Similarly, with $30 \mathrm{fb}^{-1}$ of data, one would expect to exclude the $\mathrm{CP}$-even (CP-odd) hypothesis with a confidence level corresponding to $2.5 \sigma(2.0 \sigma)$ using the $H \rightarrow \tau \tau$ channel if the Higgs boson mass is $120 \mathrm{GeV}$. After establishing that the dominant coupling is Standard Model-like, one can still test for a small CP-even anomalous coupling, since interference terms modify the $\Delta \phi_{j j}$ distribution. With $30 \mathrm{fb}^{-1}$ of data, the expected error on the anomalous CP-even coupling is $\sigma\left(g_{5 e}^{H Z Z}\right)=0.11$ if $M_{H}$ is $160 \mathrm{GeV}$, or $\sigma\left(g_{5 e}^{H Z Z}\right)=0.24$ if it is $120 \mathrm{GeV}$. These figures neglect systematic uncertainty in the background; adding a $10 \%$ background uncertainty leads to an additional error of about 0.02 in the measured coupling.

\section{COUPLINGS TO GAUGE BOSONS AND FERMIONS}

The most model-independent observable sensitive to the Higgs couplings is the total rate, $\sigma \times B R$, for each final state. In the case of $H \rightarrow W W$, the event selection assumes that the Higgs boson is CP-even and spin 0; the cut on the dilepton angle in the transverse plane exploits spin correlations to suppress the background. As shown in the last section, it is possible to verify this assumption through an analysis of $\Delta \phi_{j j}$ in Weak Boson Fusion events. Figure 2 shows the expected uncertainty on $\sigma \times B R$ for several channels [13]. For $M_{H}<150 \mathrm{GeV}$ (left), the error in the rate ranges from $30 \%$ to $100 \%$. For channels that can be seen in the mass range $110<M_{H}<190 \mathrm{GeV}$, the error is smaller.

By assuming that there is only one Higgs boson, it is possible to extract a measurement of relative branching ratios such as $\Gamma_{Z} / \Gamma_{W}$. The $H \rightarrow W W$ channel is used as the normalization since it is measured with the smallest error. With $30 \mathrm{fb}^{-1}$ of integrated luminosity, these ratios of partial widths can be measured with an accuracy better than $60 \%$ for $M_{H}>120 \mathrm{GeV}$, with a precision better than $35 \%$ for some masses.

Assuming that there are no extra particles or strong couplings to light fermions, it is possible to obtain estimates of the ratios of Higgs couplings. The cross-sections and 

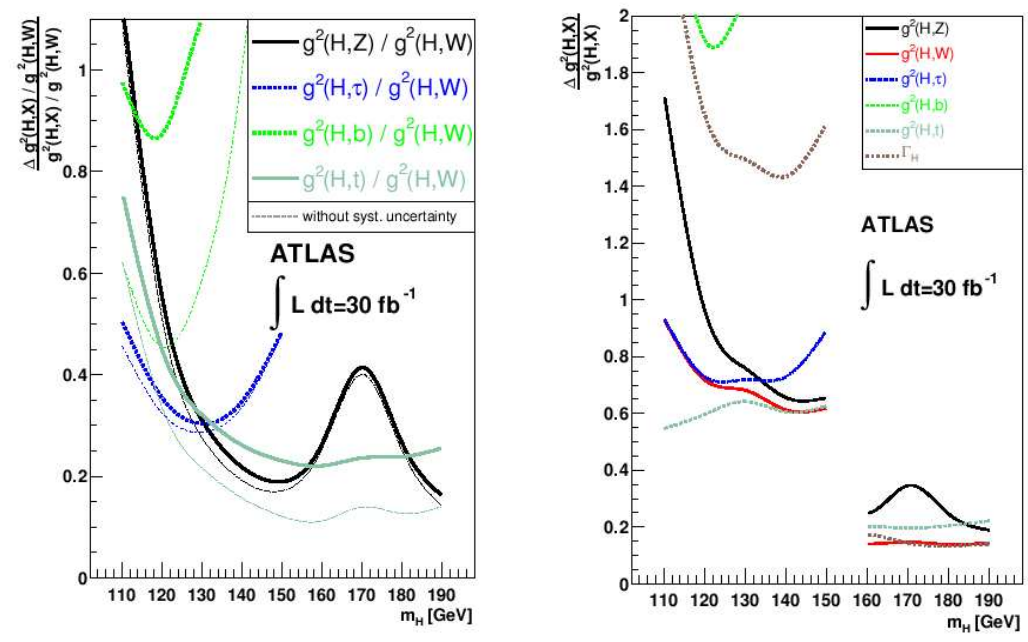

FIGURE 3. The expected error on the measurement of the relative (left) and absolute (right) couplings of the Higgs to gauge bosons and fermions after collecting $30 \mathrm{fb}^{-1}$ of integrated luminosity. The discontinuity at $M_{H}=150 \mathrm{GeV}$ is due to a change in the assumption about the sum of all branching ratios. For $M_{H}<150 \mathrm{GeV}$, decays to $b$ and $\tau$ are included in the sum; for $M_{H}>150 \mathrm{GeV}$, they are not.

branching ratios are proportional to the squares of the couplings, and the proportionality constants are taken from a theoretical calculation. The left plot of Figure 3 shows the expected relative error on the ratios of Higgs boson couplings after $30 \mathrm{fb}^{-1}$ of integrated luminosity. The uncertainties range from about $20 \%$ up to $100 \%$.

Finally, with the additional assumption that the sum of visible branching ratios is the same as in the Standard Model up to an error equal to the sum of undetected Standard Model branching ratios, the absolute couplings of the Higgs boson to the $Z$, the $W$, and the $\tau$ can be measured. The error is less than $100 \%$ for $120<M_{H}<200 \mathrm{GeV}$, as shown in the right plot of Figure 3.

\section{REFERENCES}

1. R. Brout and F. Englert, Phys. Rev. Lett. 13, 321-323 (1964)

2. P. W. Higgs, Phys. Lett. 12, 132-133 (1964)

3. P. W. Higgs, Phys. Rev. Lett. 13, 508-509 (1964)

4. LEP Working Group for Higgs boson searches, Phys. Lett. B565, 61-75 (2003)

5. Tevatron New Phenomena Higgs Working Group, arXiv:0808.0534 [hep-ex] (2008)

6. ATLAS Collaboration, G. Aad et al., "The ATLAS Experiment at the Large Hadron Collider," JINST 3:S08003,2008.

7. ATLAS Collaboration, "Expected Performance of the ATLAS Experiment, Detector, Trigger and Physics," CERN-OPEN-2008-020, Geneva, 2008, to appear.

8. The ATLAS Collaboration, "ATLAS Detector and Physics Performance," CERN/LHCC 99-15 (1999)

9. C. N. Yang, Phys. Rev. 77 242-245 (1950)

10. C. P. Buszello et. al, Eur. Phys. J. C32, 209-219 (2004)

11. R. N. Cahn and S. Dawson, Phys. Lett. B136, 196 (1984)

12. C. Ruwiedel et al., Eur. Phys. J. C51, 285-414 (2007)

13. M. Dührssen, "Prospects for the Measurement of Higgs boson Coupling Parameters in the Mass Range from 110-190 GeV," ATLAS Note ATL-PHYS-2003-030 (2003) 